\title{
Optics and \\ Measurement 2012 \\ toptec
}

Hotel Ještěd, Liberec CZ,

\author{
16.-18. October 2012
}

www.toptec.eu

The conference focuses on processes and technology connected to optical elements and systems creation. It includes design, manufacturing, measurement and testing. It should help to bring an overview of projects being solved, in the above mentioned field. Its aim is to provide the organizational backup for meeting research workers, industry experts, postgraduates and students of final years at universities who are interested in field of optics manufacturing and measurement technology. The conference should contribute to making closer contacts and sharing experience.

The main topics of the conference are:

- Classical way of manufacturing glass, plastic, metal and ceramics optical elements, spheres, flats, aspheres

- Nonclassical way of manufacturing, polishing using MRF, IBF, Plasma, Laser, etc.

- Methods and systems for optics measurement, measurement methods employing optical principle

- Thin film layer deposition

- Fine mechanics

- Optical design, simulation, experimental evaluation

- Special optics, RTG, HOE, Diffractive, Light guide, Crystal optics, etc.

- Optics for lasers, optics for space and radiation hard optics

\section{Conference is organized by}

Research Centre TOPTEC, Institute of Plasma Physics AS CR

\section{Steering Committee}

Tomáš Vít

Vít Lédl

Dana Pekařová

Jana Kovačičinová

\section{Sciences Committe}

Prof. Dr.-Ing. Rolf Rasher

Technologie Campus Teisnach,Technologiecampus 1, 94244 Teisnach

Prof. Dr.-Ing. Christine Wünsche

Technologie Campus Teisnach,Technologiecampus 1, 94244 Teisnach

prof. Dr. Wolfgang Osten

Universität Stuttgart, Institut für Technische Optik, Pfaffenwaldring 9, D-70569 Stuttgart

This is an Open Access article distributed under the terms of the Creative Commons Attribution License 2.0, which permits unrestricted use, distribution, and reproduction in any medium, provided the original work is properly cited. 
prof. RNDr. Antonín Mikš, CSc.

ČVUT Praha, Katedra fyziky, Thákurova 7, 16629 Praha 6

Ing. Marek Škereň, Ph.D.

Fakulta jaderná a fyzikálně-inženýrská, V Holešovičkách 2, 18000 Praha 8

doc. Ing. Ladislav Pína, DrSc.

Fakulta jaderná a fyzikálně inženýrská, V Holešovičkách 2, 18000 Praha 8

Doc. RNDr. Miroslav Miler, DrSc.

Ústav fotoniky a elektroniky AV ČR, Chaberská 57, 18251, Praha 8 - Kobylisy

prof. RNDr. Pavel Tománek, CSc.

VUT, Fakulta elektrotechniky a komunikačních technologií, Technická 2848/8, Žabovřesky,

61600, Brno, Česká republika

Prof. Ing. Helena Jelínková, DrSc.

Fakulta jaderná a fyzikálně-inženýrská, V Holešovičkách 2, 18000 Praha 8

Dr. Valery I. Skomorovskiy

Institute of Solar-Terrestrial Physics, Department of Solar Physics, Russia

doc. RNDr. Petr Heinzel, DrSc.

Astronomický ústav AV ČR, Fričova 298, 25165 Ondřejov

prof. Ing. Václav Kopecký, CSc.

TUL, Studentská 2, 46117 Liberec

prof. Ing. Pavel Fiala, CSc.

Fakulta jaderná a fyzikálně-inženýrská, V Holešovičkách 2, 18000 Praha 8

doc. Ing. Jiři Novák, Ph.D.

ČVUT, Fakulta stavebni, Thákurova 7, 16629 Praha 6 - Dejvice

\section{Scientific Editors:}

Doc. Ing. Miroslav Šulc, CSc.

Technical university of Liberec

prof. Ing. Václav Kopecký, CSc

Technical university of Liberec

Ing. Vít Lédl, Ph.D.

Institute of Plasma physics ASCR, TOPTEC

Mgr. Radek Melich, Ph.D.

Institute of Plasma physics ASCR, TOPTEC

Ing. Marek Škereň, Ph.D.

Faculty of Nuclear Sciences and Physical Engineering, CTU

\section{Sponsors}

Meopta - optica, s.r.o. (www.meopta.com)

Measurement Technic Moravia (www.mt-m.eu)

LAO - průmyslové systémy, s.r.o. (www.lao.cz)

CRYTUR, spol. s r.o. (www.crytur.cz)

Zeeko Ltd (www.zeeko.co.uk)

OptoTech Optikmaschinen GmbH (www.optotech.de) 